\title{
FILOSOFÍA COMO «APROXIMACIÓN INFINITA». CONSIDERACIONES A PARTIR DE LA «CONSTELACIÓN» DEL PRIMER ROMANTICISMO ALEMÁN ${ }^{1}$
}

\author{
PHILOSOPHY AS "INFINITE APPROXIMATION". \\ ON THE “CONSTELLATION” OF EARLY GERMAN ROMANTICISM
}

\section{Manfred Frank}

\begin{abstract}
RESUMEN
Este trabajo presenta las ideas filosóficas fundamentales de primer romanticismo alemán, interpretándolo como una corriente contrapuesta al idealismo alemán.

PALABRAS CLAVE: Romanticismo, idealismo, filosofía.
\end{abstract}

\begin{abstract}
This text presents the main philosophical ideas of the Early German Romanticism as a philosophical view opposed to German Idealism.

KEYWORDS: Romanticism, Idealism, Philosophy
\end{abstract}

Todo el mundo, por lo menos en el ámbito de la denominada «Historia de las Ideas», cree tener un juicio propio sobre el primer romanticismo alemán. Ya sea porque en él se ve una cumbre de la cultura europea, cuya fuerza y productividad en los más diversos campos sólo se podría comparar, a lo sumo, con las de la Atenas clásica (Henrich 1991, 217 y s.); ya sea porque en él se identifica el prototipo del «particular camino alemán» en el curso de la Modernidad y porque en él se data perfectamente el comienzo del trágico destino que se extiende «de Schelling a

\footnotetext{
${ }^{1}$ Manfred Frank, „Philosophie als ,unendliche Annäherung'. Überlegungen im Ausgang von der frühromantischen ,Konstellation“", en: Auswege aus dem Deutschen Idealismus, Frankfurt am Main: Suhrkamp Verlag, 2007, pp. 67-87. C Suhrkamp Verlag Frankfurt am Main. Traducción de David Hereza Modrego.
} 
Hitler» (Lukács 1954; en relación a las lecciones de metafísica de Heidegger del año 1941, cf. Köhler 1999).

Pero en realidad el primer romanticismo, por lo menos el filosófico ${ }^{2}$, es lo desconocido por excelencia en los archivos de la Historia de las Ideas, y no sólo en los de la «oficial». Esta situación no se debe exclusivamente a que las fuentes, como el sistema esbozado por Hölderlin en Juicio y Ser (mayo de 1795), los Estudios sobre Fichte de Friedrich von Hardenberg (de septiembre de 1795 hasta julio de 1796), o los Años de aprendizaje filosófico de Friedrich Schlegel (desde agosto de 1796) hayan sido hallados y/o críticamente editados tan solo desde hace algunas décadas; faltaba, sobre todo, una perspectiva sistemática de estos documentos, transmitidos en su mayoría de forma fragmentaria.

Sólo el «análisis de constelaciones» [Konstellationsforschung] fundado por Dieter Henrich y sus discípulos ha cambiado radicalmente la situación de la investigación desde los años 90 (cf. Mulsow/Stamm 2005). Este método hermenéutico se puede caracterizar de la siguiente manera: trata de seguir los pasos de la paulatina elaboración de pensamientos y presuposiciones filosóficas de numerosos autores a partir del intercambio de ideas que se haya podido dar entre ellos dentro de un espacio de tiempo rico en acontecimientos relevantes. En este caso, dicho periodo es el tiempo, extraordinariamente fructífero filosóficamente hablando, transcurrido en Jena entre la publicación del Ensayo de una nueva teoría de la capacidad bumana de representación (1789) de Carl Leonhard Reinhold y las primeras reacciones significativas ante la exposición de Johann Gottlieb Fichte de su Doctrina de la Ciencia (1794/95; para ello, Frank 1998). Mediante este método de análisis de «constelaciones» existe la posibilidad de completar, como en un puzle, pensamientos de un autor, transmitidos de forma fragmentaria, mediante pensamientos de otro autor (hasta el momento desconocidos o desatendidos), cuya formación o convicciones hayan seguido (o hayan podido seguir) una línea muy similar. Por fortuna, este es el caso de Hölderlin, Friedrich von Hardenberg y Friedrich Schlegel, quienes desde el comienzo de 1790 estudiaron directamente con Reinhold o estuvieron al corriente, mediante un tercero (especialmente Niethammer), de la situación de la discusión dentro del círculo de estudiantes formado en torno a él.

Un conocimiento minucioso de dicha discusión es especialmente importante para nosotros, ya que logra contraponerse a la imagen que se ha hecho en la

\footnotetext{
${ }^{2}$ En él incluyo la obra filosófica de Hölderlin y su círculo por motivos de semejanza en la estructura y en la historia de su creación.
} 
Historia de la Filosofía del proceso de reelaboración de la filosofía kantiana y del surgimiento del Idealismo alemán. Esta discusión comenzó con la convicción de Reinhold según la cual, a la filosofía de Kant le faltaba un principio supremo a partir del que se pudiese hacer comprensible y unitaria su desmembración, no sólo en principios irreducibles entre sí, sino además en tres Críticas. Sin embargo, entre los estudiantes de Reinhold pronto comenzaron a surgir dudas en torno a este programa fundamentalista; dudas que luego desembocarían en el intento de Fichte de superar los principios filosóficos de Reinhold. En cierto modo, aquellas posiciones contrarias al intento fundamentalista expresan fidelidad a Kant y muestran un camino de cómo podrían haber sido evitadas las consecuencias de un retorno a la metafísica; camino que luego seguiría, con gran repercusión, el Idealismo alemán.

Normalmente, al denominado «primer romanticismo» se le adjudica el puesto de un curioso subgrupo del Idealismo alemán. Desde hace tiempo he negado esta clasificación y he propuesto la siguiente diferenciación: «Idealista» quiere decir una forma de pensar, que reduce las estructuras de la realidad [Wirklichkeit] a las capacidades del espíritu [Geist] o — de forma inversa— que deduce éstas a partir de la evidencias aceptadas de un sujeto. «Primer romanticismo» quiere decir, por el contrario, la convicción según la cual el sujeto mismo y la conciencia, mediante la que se conoce, se basan en una presuposición de la que no se tiene experiencia alguna. Un indicio relativo a esta idea es la afirmación de Schleiermacher de un «fundamento transcendente» de la autoconciencia que como tal no debería llamarse «transcendental». Éste no puede ser expuesto adecuadamente en la conciencia de ninguna forma, sino sólo ser inferido a partir de su constitución, de otra forma inexplicable. A partir de esta consideración se puede trazar la línea de un realismo ontológico que conecta las primeras ideas filosóficas de Hölderlin y su círculo con las de Friedrich von Hardenberg y Friedrich Schlegel. Por otra parte, gracias al análisis de constelaciones, sabemos que éstas surgieron a partir de las objeciones de carácter fundamentalmente escéptico, que al inicio de 1790 aparecieron dentro del círculo de estudiantes de Reinhold. Esto se relaciona con el gran aprecio de las Bellas Artes característico de todo el movimiento. Si, en contraposición a la famosa sentencia de Hegel (Hegel 1995, 56 y s.), «lo supremo» es inaccesible a la reflexión, sólo el arte, mediante el inagotable contenido de una obra estética, puede poner de manifiesto dicha inaccesibilidad.

Desde el momento en que podemos determinar lo propio del «primer romanticismo» filosófico de forma más clara que generaciones anteriores, se iluminan también otras rastros de la tradición que, partiendo de Kant y pasando 
por el «primer romanticismo», llega hasta los transcendentalistas americanos (véase el trabajo, divulgativo pero exhaustivo, de Menand 2001). Si el «absoluto» no se deja atrapar por ningún pensamiento definitivo, éste deja de ser pertenencia de la reflexión y pasa a ser una idea en sentido kantiano. Aspiramos continuamente a ella, pero nunca podemos exponerla «demostrativamente». Aquello que dice la famosa frase de Novalis (de 1797): «Buscamos por todas partes lo incondicionado, y siempre encontramos sólo cosas» $3^{3 *}$ (NS II, 412, nº 1).

Es en torno a mediados del siglo XIX cuando Ralph Waldo Emerson transmitió el conocimiento del primer romanticismo, acabado con cierta fortuna mediante su propia inspiración, a un joven miembro del «Transcendental Club» en Nueva Inglaterra, el cual, desde entonces, ha permanecido vinculado a la idea de la filosofía como aproximación infinita a la verdad, en tanto que «verdad» es entendida como la exhaustiva comprensión («representación») de la realidad en la forma de la teoría. Estoy hablando de Charles Sanders Peirce, quien acuñó la expresión «in the long run» (que viene a decir que el run era realmente tan long que podía durar ad kalendas graecas).

Con ello, Peirce se vinculó a un pensamiento central de la filosofía del primer romanticismo; pensamiento que, por ejemplo, Friedrich Schlegel expresó al

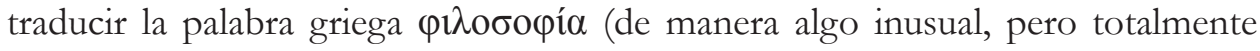
acertada) como «nostalgia [Sehnsucht] $]^{{ }^{*}}$ de lo infinito» (KA XVIII, 418, Nr. 1168; cf. 420, Nr. 2000 [en el original de Schlegel en cursiva]). Esta traducción no toma

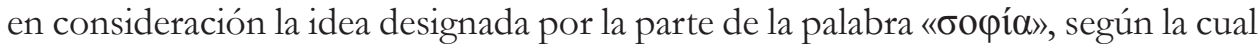
se llega a un estado intelectual en el que el alma se abre a la verdad. Ya que no se llega nunca a tal momento, el amor por la sabiduría se transforma en una nostalgia eternamente insatisfecha. Del mismo modo, Novalis escribió que su vocación se llamaba como su prometida: Sofía (Carta a Friedrich Schlegel del 8 de julio de 1796). Igual que el amor hacia ella — «filo-sofía», dice, es «el alma de mi vida, la llave de mí mismo» (NS IV, 188, líneas 8-11)— la filosofía es también una pasión

\footnotetext{
${ }^{3}$ Novalis hace un juego de palabras en alemán que es imposible verter al español. «(Un)bedingt» [(In)condicionado] tiene relación con la palabra «Ding» [cosa] (N. del T.).

${ }^{4}$ No es inútil recordar que la palabra «Sehnsucht» no es filológicamente equiparable a nuestra «nostalgia» («dolor por el pasado») — si bien el contenido semántico de las dos palabras actualmente sea casi el mismo- Por eso, en la traducción de este palabra por «nostalgia», se cancela la posibilidad al lector español de entender algo que el lector alemán atento sí puede apreciar y al que los románticos, como bien desarrolla Frank, se remiten. Lo que «Sehn-sucht» designa es, principalmente, una «búsqueda» [Suche], una «acción» y no meramente un «estado anímico» (N. del T.).
} 
irrealizable (Sartre diría: una pasión inútil [Sartre 1943, 708]); y esto por motivos que pocos han analizado tan profundamente como Novalis.

Con 24 años —atrapado dentro del engranaje jurídico de la administración del distrito de Tennstedt (y salvando sólo tres horas diarias de la dura jornada laboral para el lujo de tales preguntas)— anota: «¿Qué hago al filosofar?». Su respuesta aún hoy continúa siendo emocionante:

Pienso en un fundamento. [...] Todo filosofar debe finalizar en un fundamento absoluto (NS II, 269, Nr. 366).

¿Por qué en un fundamento absoluto? Porque uno relativo debería encadenar una serie de fundamentos sucesivos que no conducirían a ningún último elemento; pero exactamente esto parece ser el resultado. Por eso continúa Novalis con la pregunta:

[Qué sucedería] si no fuese dado aquel [fundamento último y absoluto], si dicho concepto contuviese una imposibilidad; entonces el impulso del filosofar sería una actividad infinita y, por lo tanto, sin fin, ya que existiría una necesidad eterna de un fundamento absoluto, que, sin embargo, sólo podría ser saciada de forma relativa, y, por lo tanto, no terminaría nunca (ibíd.)

Desde octubre de 1790 hasta octubre de 1791, Novalis siguió en Jena las lecciones de Reinhold y se familiarizó con la discusión del círculo formado en torno a él ${ }^{5}$. La aportación de Carl Leonhard Reinhold a la historia de la filosofía consistió en la fundación de la llamada Filosofía Elemental. Su idea principal es que podemos fundar en un fundamento último la búsqueda del saber (búsqueda hasta el momento febril e incierta); y así traduce, también de forma totalmente correcta, la palabra griega $\varphi \imath \lambda o \sigma o \varphi i ́ \alpha$. Al descubrimiento de este fundamento lo denominó —de forma algo exagerada— «lo único que es necesario a la humanidad». En 1789, en la segunda edición ampliada de su libro sobre Spinoza, Jacobi describía del siguiente modo el problema (cuya solución pretendía haber hallado): si —en una tradición respetable (y aún hoy viva)— describimos el saber como creencia

\footnotetext{
${ }^{5}$ En torno al Año Nuevo volvió a Jena (algunos datos indican que estuvo otra vez allí en febrero). Para los contactos de Novalis con el círculo estrecho de Reinhold y de Schmid, véase Weiss (1991).
} 
fundamentada, caemos en un regreso al infinito (cf. Jacobi 1789, 424 y ss., 430 y ss.). Fundamentamos nuestras pretensiones cognoscitivas en proposiciones que expresan un saber sólo bajo la condición de que sean fundamentadas en proposiciones que expresen un saber, etc. Este regreso sólo puede terminar en una proposición que valga como «in-condicionada»; es decir: que no dependa de una condición superior. Una proposición de tal tipo debería poder ser aceptada sin más como válida y así «una fundamentación no sería ni necesaria ni posible». Por lo tanto, ésta debería ser evidente, ya que «evidente» significa (literalmente): aquello que se ve por sí mismo.

Reinhold, como digo, creía haber encontrado una proposición de tal tipo. La llamó «principio de la conciencia». A partir de él deberían poder ser explicadas otras proposiciones con pretensión de verdad, mediante deducción lógica o analíticamente. Con «analíticamente» entendía Reinhold más o menos lo mismo que ha dado a la filosofía analítica actual su nombre: aquello que resulta de la comprensión del significado de las expresiones utilizadas (junto con la de sus conectores proposicionales) ${ }^{6}$.

Entre los alumnos de Reinhold pronto surgieron dudas sobre este proyecto, que en 1794 parecía consumarse en la filosofía fichteana del Yo absoluto. Tales dudas afectaban a tres puntos diferentes: En primer lugar fue puesto en discusión que un sistema de convicciones en general se pudiese apoyar en una evidencia, pues las evidencias son vivencias privadas de la conciencia. Apelando a éstas no puede ser explicada la formación de un consenso intersubjetivo; y, sin embargo, dicho consenso forma un criterio de aquello que llamamos «saber». Además de esto, si se analiza más exactamente, estas vivencias privadas no se pueden diferenciar claramente de las «pretensiones del sentido común» (Niethammer 1795). Y éstas, usualmente, se fundamentan en las denominadas «intuiciones»; es decir, en la necesidad de creer en ellas. Las proposiciones que expresan una

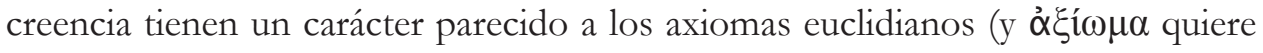
decir exactamente eso: algo en lo que se cree), de tal forma que si fuese posible probarlas, perderían inmediatamente su estatus de principios supremos, pues que una proposición encuentre su fundamentación en otra significa que no es la más alta. Así, sin embargo, la fundamentación del saber se transforma en artículos de

\footnotetext{
${ }^{6}$ La Filosofía Elemental de Reinhold y los argumentos de sus críticos más importantes son expuestos de forma detallada en Henrich (2004) y Frank (1998b). Un libro estándar sobre la Filosofía elemental: Bondeli (1995). Sobre la situación y el Philosophische Journal editado por Niethammer, Stamm (1992)
} 
fe. Novalis dirá: «es un producto de la imaginación aquello en lo que creemos sin poder reconocerlo según su naturaleza y la nuestra» (NS II, 273, Nr. 562, Línea 16 y ss.). Pero la objeción más seria y más exitosa fue la tercera: el principio supremo de Reinhold no se sostiene por sí mismo, más bien presupone otras proposiciones para su fundamentación, proposiciones que supuestamente se siguen de él.

Todo esto conllevaría terribles consecuencias para una filosofía con la pretensión de fundarse en un único principio. Y de hecho, el impulso crítico de Novalis sólo es comprensible a partir de la familiaridad estrecha con los críticos de aquella filosofía que surgieron en el círculo creado en torno a Reinhold. Menciono como representantes tres nombres: Carl Christian Erhard Schmid, antiguo preceptor de los Hardenberg y persona de confianza en comprometidas situaciones vitales; Johann Benjamin Erhard, revolucionario jacobino, a quién Novalis ayudó mediante la mediación de su tío, Karl August von Hardenber (más tarde canciller del Estado prusiano); y, finalmente, Carl Forberg, quien provocó la famosa «disputa del ateísmo» en Jena por la cual Fichte fue expulsado de la Universidad y el cual entabló un importante diálogo con Novalis durante el período de escritura del fragmento antes citado.

Consideremos, en primer lugar, algunas consideraciones a propósito de la influencia que pudo haber ejercido sobre Novalis la Psicología empirica de Schmid; un conjunto de lecciones que Novalis pudo haber seguido en 1791 en Jena - lo cita ocasionalmente (NS III, 356, Nr. 524; cf. Komm. 943)—, o de las que podía haber poseído una copia (ibíd., 1009, Nr. 81). Haciendo referencia a Crusius, Schmid criticó de forma áspera, pero objetiva, el punto partida de Reinhold, a saber, el concepto de representación como termino elemental de la filosofía (Primera parte, \IX y ss.). El concepto «representación» sería inapropiado como principio deductivo de la filosofía ya que éste sólo es obtenido por medio de la abstracción a partir de una pluralidad de sucesos o actos psicológicos ${ }^{7}$. Llevar a cabo tales deducciones a partir de un concepto genérico obtenido de tal forma no tiene mucho misterio, ya que la circularidad es obvia: obtengo mediante sucesos

\footnotetext{
${ }^{7}$ Schmid correlaciona estas vivencias o actos con las correspondientes facultades o fuerzas; pero también entiende éstas como una pluralidad irreducible: «La pluralidad de los fenómenos del ánimo nos lleva a la consideración de una pluralidad de capacidad y fuerzas, así como a la condición de su determinada posibilidad y de su existencia» (Schmid 1791, 158, \ VII).
} 
particulares via abstractionis aquello a partir de lo cual luego pretendo deducirlos (Schmid 1791 I, 18 y ss. [S VI de la Introducción]) ${ }^{8}$.

Además, aquello que cae bajo un concepto no es de ninguna forma contenido en él in nuce, como una parte (véase ya en Kant, KrV B40). Quien, por ejemplo, entiende el concepto genérico de «derecho», no sabe nada sobre la práctica jurídica actual, aunque ésta sí que esté contenida, como especie, dentro del concepto genérico. $\mathrm{O}$ quien entienda correctamente el concepto de «mamífero», no tiene por qué saber nada de los marsupiales?. A partir del concepto genérico no está dada a priori la especificación. Por ello, sabiamente, Kant renuncia a decir que la «representación» sea un principio, y menos aún un principio deductivo, si bien él mismo mostró que todos los conceptos de las funciones y afecciones mentales caen bajo él (cf. KrV A320/B376 y ss.; además KrV 676 y ss. —referencias que Schmid cita en su Psicología empírica: I, $\ X, 161-)$. Pero Reinhold declaró que realmente el «principio» de su Filosofía Elemental, el de «representación», era un «concepto genérico» ${ }^{10}$; al mismo tiempo, afirmó que en la filosofía era necesario proceder mediante la diferencia especie-género, es decir, inferir, de forma ascendente, los conceptos como especificaciones siempre más altas hasta que (dice él) «se diese con algo indisoluble» (Reinhold 1790, 16; cf. 358). Pero, obviamente, a partir de un concepto tal sólo se puede derivar aquello que previamente ha sido abstraído, es decir, aquello que ya había sido conocido con anterioridad; y esto no es muy complicado de llevar a cabo. A algo parecido llegó en 1790 el profesor de Novalis en Leipzig, Karl Heinrich Heydenreich, el cuál reprochó a Reinhold, en vistas a su principio supremo, su concepto de «representación»:

\footnotetext{
${ }^{8}$ En relación a Crusius, véase también el \XII de la primera parte (164): «[...] que todo lo que deba ser explicado se pueda después extraer de nuevo». Aquí está el origen histórico de la mofa de Nietzsche: Kant responde a la pregunta de la posibilidad de los juicios sintéticos a priori con la respuesta: «en virtud de una facultad» ( $11^{\circ}$ aforismo de Más allá del bien y del mal, en KSA 5, 23-25). Para el contexto completo, cf. Frank (1998, 275 y s.).

${ }^{9}$ Véase la famosa anotación de Kant en el $\$ 61$ de la KU B268 y s.: «Pues cuando se aduce, por ejemplo, la estructura de un ave, la cavidad en sus huesos, la disposición de sus alas para el movimiento y de la cola para servir de timón, etc., se dice que todo esto es harto contingente en la naturaleza según el mero nexus effectivus, sin recurrir subsidiariamente a un peculiar tipo de causalidad cual es la de los fines (nexus finalis); es decir, que la naturaleza considerada como simple mecanismo hubiera podido configurarse de mil maneras diferentes sin dar con la unidad conforme a tal principio y, por lo tanto, que no cabe esperar el menor fundamento a priori de esta unidad sino fuera del concepto de naturaleza y no en el mismo».

${ }^{10}$ Reinhold (1790, I, 117, [passim])
} 
La representación y la capacidad de representación no son lo prius, sino lo posterius y no pueden, de ningún modo, servir como premisas para la ciencia (en: Reinhold 1790, 427 y s.)

Con estas ideas confrontará las suyas Novalis, quien fue alumno tanto de Heydenreich como de Schmid. Lo hará en algunas partes de las anotaciones que empiezan más o menos en el número 466 y que prosiguen en numerosas páginas posteriores. Éstas empiezan con ciertas consideraciones en torno a la posibilidad de que una «esfera», que contenga «esencia»y «propiedad» como los correlatos más elevados del absoluto (NS II, 251, línea 14 y s.; ya en Nr. 444, 21, línea 20 y ss.), pueda ser entendida realmente como «el género supremo — el género de todos los géneros, o el género absoluto como tal» (línea 23-25)—; y terminan con la idea de que la progresiva abstracción hasta el género supremo, o mejor, la búsqueda de lo absolutamente primero en una cadena de deducciones es un «sinsentido»: «es una idea regulativa» (254, línea 11 y s.). En el texto, el centro de atención gira en torno a un argumento identificable en cuatro partes:

1. Para que algo pueda ser definible (o, como dice Reinhold, «absolutamente autodeterminado ${ }^{11}$ ), necesita para su limitación un género menor y uno mayor. La última exigencia es, sin embargo, en el caso del género supremo un sinsentido: no hay en ella «una característica general y específica» ( $\mathrm{Nr}$. 445, 243, línea 6 y ss. $)^{12}$. De esto se sigue que:

2. No se pueda aspirar a alcanzar el fin de una cadena argumentativa:

$\mathrm{Al}$ final, cada género presupone ${ }^{13}$, de forma completamente necesaria, algo mayor que lo abarca —un espacio- y si esto es así, el género supremo es un Nonens. [...] Los conceptos de género, especie y singular tienen sólo un uso regulativo y clasificatorio — ninguna realidad en sí, pues entonces deberían ser infinitos-. No debemos seguir esta idea, pues entonces caemos en el lugar del sinsentido (Ibíd., 251 y s.).

\footnotetext{
${ }^{11}$ En contra de este concepto tiene Novalis una objeción más profunda que, de hecho, se dirige más bien contra Fichte y da un golpe central a su idealismo: «una actividad capaz de determinarse a sí misma es un sinsentido [Unding]; toda actividad determinada presupone de forma necesaria un límite, algo existente» (NS II, 242, Nr. 444, Línea 7-10).

12 Véase el párrafo completo, que pone a prueba el concepto de «Definición» — como «conteniendo el concepto objetivo de una cosa» (262, Nr. 526) — ante la pretensión de la «Teoría». ${ }^{13}$ Véase NS II, 251, línea 2 y ss.: «Si hablamos del géneros, qué entendemos por ellos: un carácter fundamental común — pero no encontramos los géneros siempre contenidos en algo que los abarca $[\dot{2} ?] \gg$
} 
3. Luego, Novalis pasa a considerar algunos de los candidatos propuestos por sus coetáneos para la identificación del género supremo: primero el concepto de «cosa» (251, línea 5 y s.; ya antes passim), luego el de «representación» (ibid.), finalmente el del «Yo» o «sujeto» (Nr. 470, 253, línea 20 y s.). Todo son rechazados. El del «Yo» porque es elemento de una contraposición, parte de una esfera y, por lo tanto, no se puede pensar como absoluto (253, línea 28). ${ }^{14}$ Tampoco tiene ninguna indulgencia con el concepto de «causa (absoluta)» (en el sentido de la parte conclusiva del Ensayo de una nueva teoría de la capacidad humana de representación de Reinhold $)^{15}$. Proyectado al «infinito», «causa» sería, de nuevo, «sólo un concepto regulativo, una idea de la razón» - no tendría sentido, pues, querer probar su validez real. Buscamos, pues, una algo absurdo [Unding]» (Nr. 476, 255, línea 12-14; véase Nr. 477, línea 25 y s.) -

4. Un argumento (añadido más tarde) recuerda directamente a Heydenreich y Schmid. Dicho argumento señala que aquello que fuese encontrado en el transcurso del método de la abstracción progresiva, es decir, el «género supremo», viviría de la realidad de aquello de lo que fue abstraído. Querer llevar a cabo deducciones a partir de un concepto de género encontrado de tal modo no sería sólo circular; sino que, al mismo tiempo, se presupondría y no se explicaría el individuo: «mediante el género nunca puedo llegar a conocer el individuo, sino mediante los individuos el género» (Nr. 567, 271, línea 17 y s.). «La esfera característica del género es la especie, o el individuo. Él existe sólo por esto último» (Nr. 513, 261).

Schmid — volviendo a él— atacó a Reinhold otra vez de forma muy eficaz, concretamente, en la recensión del escrito Sobre el fundamento del saber filosófico de principios del abril de 1792 (publicado en la $A L Z^{16}$ de Jena). Allí muestra que Reinhold se equivoca cuando cree que el conjunto de las facultades descritas

\footnotetext{
${ }^{14}$ Véase NS II, 256, Nr. 478, línea 3: «Yo’ es expresión del singular que Enjuicia, “que tiene representaciones"».

${ }^{15}$ Véase para ello Frank (1998), Lección 11, 268 y ss.; segunda mitad de la lección 15, 418 y ss.; lección 18, 485 y ss.

${ }^{16}$ Frank se refiere con estas siglas a la Allgemeine Literatur-Zeitung, revista publicada en Jena entre los años 1785-1803 (después en Halle hasta 1849) a cargo de, entre otros autores, Ch. G. Schütz, quien desde el principio quiso hacer de ella un organismo de apoyo en la difusión de la «filosofía crítica». La revista consiguió reunir a los mejores autores de la época: Kant, Schiller, Reinhold, Fichte, Humboldt, etc. (N. del T.).
} 
por Kant se pueden reducir a un solo principio. Un paso de esta argumentación, decisivo para Novalis, dice que Reinhold, en vez de deducir conclusiones a partir del principio de la conciencia como proposición fundamental, como mínimo presupone, más bien, algunas de ellas como ya válidas de forma tácita («que el principio de la conciencia no ha hecho lo más mínimo para demostrar aquellas proposiciones, que [más bien, al realizar dicha deducciones,] se han tomado en consideración otras proposiciones y tácitamente se ha recurrido subsidiariamente a ellas» [Schmid 1792, 57 y s.]). El principio de la conciencia es, brevemente, el siguiente: «En la conciencia, la representación es referida, por el sujeto, al sujeto y al objeto, y diferenciada de ambos». El agente propio en todos los pasos que traza esa fórmula es, por consiguiente, la autoactividad del sujeto. Esta actividad (del mismo modo que la casualidad), Reinhold pretende haberla deducido a partir del análisis del concepto de representación. Generalizando la objeción, ésta dice: Reinhold no ha deducido las consecuencias a partir del principio, sino que las ha presupuesto.

Schmid encuentra otro error en la argumentación de Reinhold, esta vez uno técnico: una deducción o derivación lógica se efectúa, normalmente, a partir de un término mayor, universal, y un término menor, singular, que es independiente lógicamente del término mayor. «Todos los gatos tienen pelo. El gato Murr es un gato. Por lo tanto, Murr tiene pelo». Ahora bien, el «principio de la conciencia» es un caso particular, no universal. Para poder llegar a las consecuencias supuestas por Reinhold, éste necesitaría apoyarse en un término mayor, el cual sólo podría haber sido deducido de forma inferencial (¿cómo si no?). Brevemente: Las derivaciones de Reinhold tienen, como tal, el carácter de una metodología hipotético-deductiva tal y como sucede en las derivaciones kantianas de las ideas (regulativas) de la Razón o en las derivaciones siempre refutables de Popper: «Lo que es tomado como hipótesis [y no, según lo dicho, como evidencia inmediata] nunca puede tener la pretensión de valer universalmente, ya que siempre queda sin determinar si se quiere aceptar como hecho indudable esta hipótesis de la experiencia, otra, o ninguna» (Schmid 1792, 59). Al final, pues, siempre queda abierto el conjunto de premisas a partir del cual se sigue (según una o más reglas generales) un explicandum; o, dicho de otra forma, éste no está suficientemente determinado mediante la existencia de ese explicandum concreto. Algo que, justamente, habían ya puesto de relieve Kant, Maimon y Aenesidemus-Schulze. 
Mucho más impactantes (dada la fuerza de repercusión que tendrán para el posterior desarrollo de la filosofía extraída del espíritu kantiano) son las objeciones de Erhard. Éstas fueron desarrolladas en el intercambio epistolar con los críticos del círculo de Reinhold, y señalan, de la forma más precisa posible, la convicción general de los mismos.

De entrada, Erhard no problematiza el hecho de que la autoconciencia sea el primer principio (o punto de partida) de la filosofía, algo que tampoco fue la idea inicial de Reinhold. Éste fue el camino que, en el verano de 1792, Reinhold se vio obligado a tomar bajo la influencia de sus críticos, entre ellos el antiguo repetent $t^{1 *}$ de Tubinga Carl Diez ${ }^{18}$. De hecho, ya en torno a 1790, Reinhold ofrecía una formulación estándar de su teoría de la capacidad de representación según la cual el sujeto es el único agente de las operaciones de las que trata el «principio de la conciencia): Es el sujeto, quien, en la conciencia, refiere la representación tanto a sí mismo como al objeto, y la diferencia, al mismo tiempo, de ambos. Si se sigue al pie de la letra esta nueva posibilidad, se llega rápidamente a la convicción de que todo debe ser caracterizado como auto-referencia de una conciencia y que lo único activo es el sujeto. En relación a este punto de partida de Reinhold, anota Novalis: «El sujeto es presupuesto en toda conciencia — es el estado, absolutamente activo, de la conciencia» (NS II, 253, línea 25 y s.). Esto también fue aceptado por Erhard; lo que, sin embargo, no concedió es un estatus epistemológicamente relevante a la autoconciencia. En una sarcástica recensión del escrito del Yo de Schelling — que amargaría y heriría al autor de tal modo que éste incluso llegaría a negar, en una réplica agresiva y poco convincente, que en ese escrito hubiese apuntado a una filosofía-fundada-en-un-principio-supremo (SW I/I, 242)—, Erhard reprocha a aquellos que especulan sobre un supuesto «Yo absoluto» el expresar de forma descriptiva aquello que es radicalmente diferente a un posible objeto de nuestra conciencia (empírica). Sólo llegamos a ser conscientes de lo determinado y, por lo tanto, de aquello que está limitado por otra cosa. Es decir, nunca podremos ser conscientes del Yo en su libertad absoluta ya que, según Erhard, un tipo tal

\footnotetext{
${ }^{17} \mathrm{El}$ «Repetent» era un joven seminaristas recién licenciado que ejercía como auxiliar de los profesores titulares de los seminarios — en este caso, del famoso Seminario de Tubinga-. Concretamente, a éste se le encargaba dar un tipo de clases llamadas repetitiones (hoy diríamos «repasos»), donde éstos podían entablar cierta relación estrecha con los seminaristas, con los que se diferenciaban poco en edad (N. del T.).

${ }^{18}$ Para ello confróntese la monumental obra de Dieter Henrich (2004, especialmente I, 388 y ss.).
} 
de conciencia agotaría la esfera completa de la conciencia (y esto quiere decir, al mismo tiempo, nuestra personalidad moral [Erhard 1796, 91]). Su presumible serabsoluto y ser-puro se apoya en su indeterminación objetiva (91) que Schelling expresó en la noción de «intuición intelectual» (90). Erhard finaliza la recensión con un hiriente mensaje:

Tanto como él [el autor] ha podido entender, el objeto real del mismo [del sistema de Schelling] es apoyado en la nada, entendida como intuición intelectual (que no merecería tan siquiera ese nombre al ser intuida con ella «la nada»). Pues el autor, en toda su investigación introspectiva, no ha podido encontrar nada a lo que el predicado «yo absoluto» se ajustase, si no es forzando un estado de ausencia de pensamiento al que se puede llegar si se inhibe totalmente el camino de la imaginación y en el cual no se tiene otro sentimiento que el de la autodeterminación. Este particular sentimiento tiene realmente algo de totalmente misterioso, porque en él no se puede diferenciar nada y una filosofía fundada en éste no puede parecer otra cosa que la descripción vital de Nadie; se puede decir todo lo posible sin peligro de tomar la responsabilidad sobre lo dicho porque, sea como sea, no se ha querido decir aquello que ha sido refutado por el otro. Sin embargo, en Alemania parece necesario fundar una filosofía que, mediante el hundimiento en la gran nada (que las sectas hindúes glorifican como el sumo bien), tuviese un principio único y una meta única, cuando lo que es realmente necesario, es que ésta debe estar fundamentada en algo más noble. Esto no puede ser otra cosa que el sentimiento de nuestra personalidad. [...] en cuanto entes morales no somos [de hecho] ningún tipo de objeto del saber, sino del actuar (90 y s.).

De ningún modo extrae de aquí Novalis estas conclusiones morales, pero sí coincide plenamente con Erhard en la idea de que «del mismo modo (...) que no hay un espacio absoluto, no hay un sujeto absoluto» (NS II, 253, línea 28 y s.). Ya en las primeras partes de los Estudios sobre Fichte, Novalis reflexionó sobre las condiciones bajo las cuales la conciencia podía tener acceso a un Ser trascendente (o «Ser originario»). La conciencia no es para él ningún tipo de autoposición, sino el (pasivo) «sentimiento» de un límite, más allá del cual debe ser aceptado algo como creencia: «el Yo no [es] en el fondo nada [...] — a él le debe ser dado todo; y, por lo tanto, la filosofía necesita siempre algo dado; nosotros nacemos [con categorías vacías] - i.e. con superficies sin contenidos. [...] Estas esperan ser rellenadas [gefüllt] — éstas no son nada sin contenido. Éstas tienen un impulso por existir, por lo tanto, por tener un contenido, pues sólo son reales [wirklich] en la medida en que tienen un contenido» (NS II, 273, línea 31 y s.; 133, línea 30; 250, línea 19 y ss.). Por supuesto, esta donación de contenido debe acomodarse a la estructura de nuestra conciencia, que Novalis entiende como reflexión, y por tanto como 
desfiguración y distorsión de lo dado («ordo inversus»). No obstante, la reflexión puede ilustrarse sobre su «esencia distorsionada» y con ello enmendarla. En las primeras páginas Novalis aún gira en torno a la idea de una «intuición intelectual», entendida de forma totalmente distinta a la de Schelling. No es entendida como plenitud del ser — plenitudo realitatis_-, sino como imposibilidad de conocer tal cosa: «el espíritu del sentimiento está fuera de ahí»; «los límites del sentimiento son los límites de la filosofía»; «el hombre siente el límite que rodea todo para él, a él mismo, la primera actividad; debe creer en ella, por muy cierto que sea su saber acerca de todo lo demás» (114, 8 y s.; ibíd., I y s.; 107, 1-3).

En el transcurso de sus Estudios sobre Fichte la función de la intuición intelectual va perdiendo cada vez más fuerza, llegando finalmente a ser rechazada en pos de un retorno a la noción de idea o postulado en sentido kantiano — algo en total correspondencia con el espíritu de Schmid y Erhard.

Pero Erhard despertó a muchos de sus coetáneos del sueño de una filosofía basada en el principio supremo mediante una duda metódica de mayor efecto:

La filosofía [dice él] que parte de un principio y en la que todo se deduce a partir de él, permanece siempre un artificio sofístico; la verdadera filosofía es sólo aquella que avance hasta el principio supremo y presente todos los otros en perfecta harmonía con él y no como deducidos de él (Carta a Niethammer del 19 de Mayo de 1794; en: Niethammer 1795, 79).

En la carta ya citada a Reinhold y a Niethammer (de julio de 1792 y de mayo de 1794), llama a este método «análisis» (sobre ello Frank 1998, lecciones 15 y 17). Expresado en términos de la escuela de Wolff (de la cual Kant es también dependiente), este método consiste en ir de lo fundamentado al fundamento; algo contrario a la deducción de Reinhold y Fichte, que sería «sintética». La filosofía, sin embargo, no puede proceder sintéticamente ya que el principio de la conciencia o aquel que expresa el Yo de Fichte no está de entrada justificado, es decir, no es algo que sea válido por sí solo. Más bien, su verdad está unida a presuposiciones que de entrada no se poseen. Éstas sólo pueden ser alcanzadas (si bien siempre sólo de forma hipotética) mediante un movimiento regresivo de lo condicionado a sus posteriores condiciones. $\mathrm{Si}$, además de esto, se acepta que dicho camino nos conduce al infinito, es decir, que, en general, no existe una última certeza, entonces se debe renunciar totalmente a la idea de una fundamentación definitiva. Así, el lugar que ocupa lo «infinito» se remplaza por la (romántica) «nostalgia» de él; y luego, en lugar de una teoría de la verdad basada en evidencias surge una que debe mostrar (como dice Erhard) las relaciones del mundo y de la conciencia en la «harmonía» más exacta posible. Por lo tanto, se trata de un tipo de teoría coherentista de la cual 
Novalis también es partidario; así, a la hora de señalar (en filosofía) el fundamento de la justificación de una convicción, no se hará describiéndolo como algo dado, sino como algo en la «relación [de todo lo singular] con el todo» (NS II, 269, línea 27). Esta teoría coherentista, continúa diciendo Novalis, aparece como la única posible que prescinda del hecho de que sea dado un «fundamento absoluto» y, por ello, que deba hacer comprensible el «fundamento propiamente absoluto [...] mediante la unión (totalización) de lo explicado / en el todo». Novalis llama a este «procedimiento», como se puede leer en la cita, «totalización» [Verganzung]. En lugar de lo incondicionado surge para los románticos la búsqueda del mismo. «Buscamos por todas partes lo incondicionado, y siempre encontramos sólo cosas» (NS II, 412, Nr. 1).

Podría mostrarse en detalle cómo reaccionó la «filosofía de un principio único» de Reinhold y Fichte a las objeciones escépticas. En 1795 apareció otra objeción surgida en el círculo de estudiantes de Reinhold, presente en el artículo de Paul Johann Anselm Feuerbach («Sobre la imposibilidad de un principio primero y absoluto de la filosofía», Feuerbach, 1795). Esta crítica ya ha sido comentada anteriormente y la hemos encontrado ya al hablar de la crítica de Schmid a Reinhold: la evidencia de un principio supremo es una pretensión injustificada que, en fondo, comparte el mismo destino de las intuiciones del sentido común. La pretensión de la evidencia de un principio tal exige una justificación a partir de razones filosóficas, que, sin embargo, es llevada a cabo mediante ideas, y las ideas son hipótesis o conclusiones a partir de la mejor explicación, no evidencias. Por otro lado, las evidencias son hechos; y los hechos sólo son individuales y, por lo tanto, sólo pueden proporcionar la premisa menor — es decir, la segunda premisa - en una inferencia válida modo ponente (tal y como lo que Kant llama «silogismo de la razón» [KrV A103]), ésta tiene la estructura M · P, S · M, entonces $\mathrm{S} \cdot \mathrm{P}$ ). El término mayor debe ser, por el contrario, un condicional universal («para

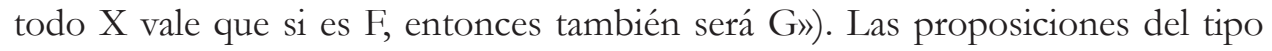
«Si...entonces...» no implican la existencia, necesaria para un principio real. Finalmente vimos también que, por otro lado, Erhard discutió la accesibilidad epistémica, es decir, la comprensibilidad del Yo absoluto de Fichte y Schelling. A esta idea de su amigo en Jena se adhirió Novalis, llegando incluso a ir tan lejos como hicieron Feuerbach y (como veremos inmediatamente) Forberg, al explicar que «lo incondicionado del saber humano» es un «non-ens», una «no-cosa», es decir, algo «imposible». (Sobre esta atrevida conclusión no se ha pronunciado nadie en la literatura secundaria sobre Novalis; allí Novalis sigue siendo un joven pánfilo «inundado de luz de luna» que nunca dejó de sentarse a la derecha de su querido 
Dios como su hijo predilecto, y el cual conoce el paraíso sólo desde arriba — como por ejemplo dice Emil Staiger, de quien procede la expresión).

Respecto a estas críticas, cabe decir que, si bien Reinhold (en una carta a Erhard del 18 de junio de 1792, recientemente publicada por Marcelo Stamm) señaló a Schmid y Diez como los autores que le hicieron modificar su idea respecto a su Filosofía elemental (Diez 1997, 911-914; véase la introducción de Marcelo Stamm, p. 898 y ss.), Diez sólo llevó a buen término las objeciones de Erhard que (como ha mostrado Henrich) le fueron conocidas mediante Niethammer.

Dada su importancia, repetimos una vez más: mediante las objeciones expuestas, el proceder de una fundamentación a partir de un principio último se transformó en una búsqueda del fundamento que, de entrada, no prevé dónde termina o si, en general, termina. Y justamente esta conclusión fue tomada en consideración por Novalis y expresada en su determinación de la filosofía como búsqueda infinitamente abierta (citada al inicio).

La filosofía kantiana llamó «ideas» a los últimos fundamentos de nuestras convicciones. Con ellas Kant entendió conceptos a los que no se les puede atribuir realidad objetiva, pero que debemos admitir para dar forma sistemática a nuestras creencias. Si un principio es una «mera» idea, entonces éste es sólo hipotético. Si, como dice Novalis, «buscásemos» la justificación de la realidad de tal principio, entonces caeríamos en «los espacios del sinsentido». El énfasis con el que pudo Novalis anotar esto, podemos observarlo en las numerosas repeticiones del calificativo «sinsentido» o «absurdo» [Unding]. Así, dice: «toda búsqueda del primer [género] es un sinsentido - es un idea regulativa» o: «[aquello que buscamos] es, sin embargo, un concepto regulativo, una idea de la razón - es absurdo dar prueba de su efectividad real» (NS II, 252, línea 6; 254, línea 11 y s.; 255, línea 12 y ss.). De esta forma, también sopesa si el «Yo» (de Fichte) no es «como toda idea de la Razón, un uso meramente regulativo, clasificatorio - con ninguna relación con la realidad» (NS II, 258, línea 18 y s.). Una idea, por lo tanto, es algo de lo que no se puede dar una determinación de su realidad empírica y, así, es imposible demostrar la realidad de una argumentación mediante una idea. Ciento cincuenta años más tarde Wittgenstein anotará que «la cadena de los principios tiene un fin». Pero no porque al final demos con una evidencia («obvia» de forma intersubjetiva), «sino porque —en ese sistema— no hay ningún fundamento» (Wittgenstein 1984, Vol. 8, 342, Nr. 301; véase 346 y s., Nr. 314; «detenerse, ahí está la dificultad»).

Novalis, sin embargo, va mucho más allá cuando, al hablar de conceptos que garantizan la unidad de nuestro sistema de creencias y principios, habla de «ficciones 
necesarias» (NS II, 179, línea 17 y ss.). Una ficción no es un descubrimiento [Findung], sino una invención [Er-findung]:

El principio supremo no debe ser algo dado, sino algo creado libremente, algo producido, pensado, para fundar un sistema metafísico universal [...] (ibíd., 273, línea 22-24). ${ }^{19}$

Esta afirmación no es sólo una conclusión muy fuerte y realmente asombrosa en el contexto del programa fundamentalista del idealismo absoluto; mediante ella se cierra de nuevo el círculo que retorna al «método del análisis» de la capacidad de representación de Erhard. Este método fue caracterizado a menudo como método de «invención», como hizo el joven Reimarus (Johann Albrecht Hinrich, hijo del famoso Samuel Reimarus), un (médico y) lógico conocido en la época, con el que Erhard tuvo un intercambio epistolar y que Novalis pudo conocer a través del libro de lógica de Johann Christoph Hoffbauer; libro al que se refiere en los Estudios sobre Fichte (NS II, 191, línea 21) ${ }^{20}$.

Como ya había señalado Wolff, mediante la forma de la invención se introducen momentos de incerteza en el proceder del análisis filosófico (Hoffbauer 1810, 23 y ss). Pero sólo el joven Reimarus, dice Hoffbauer, llegó en el \$259 de su Doctrina de la Razón a las últimas conclusiones:

Inventar algo, dice [éste], quiere decir: llegar al conocimiento de lo hasta el momento desconocido mediante el pensar propio. [...] El inventor no llega a aquello que encuentra mediante la mecánica aplicación de reglas, mediante las cuales éste puede asegurar ya de antemano lo buscado; $[. .$.$] en segundo lugar no inventamos$ aquello que es resultado de la aplicación mecánica de una regla. De ahí que se pueda decir: «inventar» se denomina al encontrar, a partir de lo conocido, lo que nos era desconocido hasta el momento, de un modo mediante el cual no sigamos meramente una regla ya conocida por nosotros (Hoffbauer 1810, 23 y s., 25 y s.). ${ }^{21}$

\footnotetext{
${ }^{19}$ Véase la caracterización posterior (de 1798/99): «Análisis es (la Divination, o) la creación artística llevada a reglas» (NS III, 434, Nr. 858). Respecto al miedo de, con dicho proceder, caer «en los espacios invisibles, y en la infinitud», así como de perderse «en el camino de la locura», en el «mal famado y falso misticismo - [en] la creencia [...] del origen de la cosa en sí», véase 442, Nr. 906.

${ }^{20}$ Véase la determinación del método analítico llevado a cabo por Johann Christoph Hoffbauer en su reelaboración del escrito premiado de 1806 Sobre el análisis en la filosofía (Hoffbauer 1810, 6-8). Hoffbauer hace referencia allí a los parágrafos 416, así como 503-505 de la Acroasis logica de Alexander Gottlieb Baumgarten (Baumgarten 1761).

${ }^{21}$ Las reflexiones propias de Hoffbauer sobre este punto son de gran interés, no sólo por el hecho de que en la redacción de sus Estudios sobre Fichte Novalis utilizase dicho autor (autor que
} 
Así, el proceder de la búsqueda analítica de un principio se aproxima a la invención, es decir, a un método que crea reglas, pero no que las sigue; método que siempre ha sido asociado a la actividad poética (y en general, al arte). Walther von Stolzing pregunta en Los maestros cantores de Núremberg: «¿Cómo comienzo según la regla?» y Hans Sachs le responde: « Ponedla, y seguidla después» (SSD 7, 239). Naturalmente, estas consecuencias no pasaron inadvertidas para el jurista, mineralogista y poeta Novalis, que con el tiempo comenzó a apreciar el dejar atrás «esa montaña escarpada de la razón pura (...) y vivir de nuevo con cuerpo y alma en el vivo país de los sentidos».

Se puede apreciar la filosofía sin tenerla como directriz y vivir sólo de ella. La matemática sola no hace soldados y mecánicos, la filosofía no hace hombres (NS IV, 321; carta de febrero de 1800 a C. August Just, alto cargo del comercio de la Turingia).

Naturalmente, esto sólo vale para un genio universal como Novalis bajo la presuposición (no aceptada por Kant), de que también los métodos de la ciencia exigen genialidad, es decir, capacidad de creación artística ${ }^{22}$.

Sin embargo, aquí no se trata de una invención de cualquier tipo. Lo que no es encontrado en la búsqueda, sino que es tomado como algo inalcanzable, es (con las palabras de Novalis) un «fundamento absoluto». Sólo éste podría dar un

ya en publicaciones previas se había pronunciado de forma similar sobre el método analítico). También en otra ocasión llegó a ser importante para Novalis el «método del análisis»: en el marco de los estudios matemáticos que emprendió en relación con su actividad de mineralogía como director de las salinas. Allí se trataba de extraer una conclusión de la relación química de los minerales a partir de la estructura de la superficie plana, calculada geométricamente, de los hallazgos (para ello, Bark 1999, especialmente 420 y ss.). En los estudios matemáticos de Bassu y Murhard (de 1798 [NS III, 115-124]) se apoya Novalis de forma determinante en una explícita paráfrasis de Maimon (en el artículo «Sobre el uso de la filosofía para la ampliación del conocimiento», publicado en el Philosophisches Journal (II/1, 1795, 1-35)) del compendio de Friedrich Murhard (Sistema de la doctrina de las magnitudes generales según su estado al final del siglo XVIII según la literatura y la historia, Lemgo: Meyer, 1798, 6 y ss.). Tomando en consideración la página 9 del compendio de Murhard determina Novalis el análisis en un sentido amplio como la «representación de lo desconocido mediante lo conocido» (NS III, 120, línea 25 y s.). Aquello que, además, Novalis aprendió del artículo de Maimon sobre el método de la filosofía será objeto de mi estudio en otro artículo.

${ }^{22}$ Hoffbauer defiende este punto de vista, expresamente contra Kant, en su tratado «Sobre el genio y la capacidad de la cabeza» (en: Hoffbauer 1810, 101-113). Sin embargo, Kant ya había denominado en la Lógica «[a]l método analítico [...] el método del hallazgo» (AA IX, nota a $\$ 117,149)$. 
fundamento a nuestras débiles vidas e inseguras convicciones. Pero un fundamento tal es, en el sentido de la palabra, una ficción: algo imaginario [Er-dichtung].

Terminaré rápidamente este esbozo con algunas frases concisas. Al determinar la búsqueda de un fundamento como «invención» se da al proceder de las artes un papel clave, a saber: éstas tienen la tarea de hacernos presentes indirectamente algo último, incomprensible mediante la fundamentación racional. Tal alto modo de comprensión es el que se tenía de las artes antes de que Arthur C. Danto anunciase el fin de la invención y del intercambio de innovaciones (Danto 1993). Olvidamos muy rápidamente lo que la expresión «religión del arte» quería decir en su tiempo y con qué sacra comprensión entendían sus productos no sólo los simbolistas (hasta Stefan George), sino incluso los provocativos expresionistas y surrealistas, o incluso Joseph Beuys. Para los autores de la teoría crítica - por encima de todos, Adorno - era el mundo ficticio del arte el lugar donde se podían salvar las últimas pretensiones de universalidad en una humanidad devastada de forma total e insalvable. Heidegger o Derrida intentaron ampliar la filosofía misma mediante el lenguaje del arte, acabando incluso en él. El mismo Wittgenstein quiso entender el Tractatus como una obra de arte literaria, en la cual lo dicho demarcase el espacio de lo indecible, que, sin embargo, era el auténtico mensaje de la obra (Frank 1999).

Y así, como «representación de lo irrepresentable» — es decir, del fundamento de nuestra vida consciente- quiso entender Novalis el arte (NS III, 685, Nr. 671). En otro lugar, anotaría:

Por su carácter mismo, no se puede pensar ninguna posibilidad de alcanzar lo inalcanzable - en cierto modo éste es sólo la sumaria expresión ideal de una serie completa, y por tanto sólo en apariencia es su último eslabón- el tipo de todo eslabón, del que todos los otros son indicios. [...]

[Por ello] las obras de artes más elevadas [...] [son] propiamente insatisfactorias — son ideales que sólo nos pueden- y deben [sollen] - gustar (en tanto que imperativos estéticos) de forma aproximativa (ibíd., 413, Nr. 745, Nr. 748).

O:

Si el carácter del problema dado es el ser irresoluble, entonces solucionamos el mismo si exponemos la irresolubilidad [como tal] (ibíd., 376, Nr. 612).

Mediante la inexpresable plenitud del sentido de una obra de arte, dice él, se nos habla (de forma alegórica, es decir, queriendo decir otra cosa de lo que se enuncia a primera vista) de aquello que no podemos hallar en la claridad y distinción del concepto, de la «paradoja de nuestra existencia» (TS II, LXXXIX, XC); «inagotable» debe ser la obra de arte: «como un hombre» (NS III, 664, Nr. 603). Así, el arte señala 
que, en verdad, aquello que se puede decir de la esencia del hombre, aquello que fundamentase nuestras frágiles convicciones, no puede ser considerado como algo que poseamos. Por ello los románticos giraron en torno al concepto de «nostalgia» -nostalgia es al final un estado en el que no se tiene nada, en el que no se posee nada (KA XVIII, 418, Nr. 1168; 420, Nr. 1200)—. Sólo podemos ir al encuentro de lo que aspiramos conseguir mediante una «aproximación infinita». ¿Quién no ve aquí una cierta similitud con la posición de Popper contra la «Hypotheses non fingo» de Newton? Especialmente cuando su obra principal tiene como lema el dístico de los Diálogos de Novalis: «las hipótesis son redes, sólo cogerá quien lance. / ¿No fue América hallada por hipótesis? / [etc.]» (NS II, 668, línea 26 y s.). La misma promesa nos da la obra de arte, la cual, justamente por eso, se muestra como siempre insatisfactoria y nos exige de forma imperativa el no llegar a expresar nada, sino a situarnos en un estado de intranquilidad: en una indeterminada aspiración hacia un fundamento, como el que desesperadamente busca y no encuentra Agatha en el Hombre sin atributos de Musil (Frank 1983).

Aproximadamente en el mismo periodo en el que Novalis anotaba estos pensamientos sobre la infinitud, es decir, sobre la imposibilidad de la filosofía como ciencia, recibió la visita de «Forberg en Jena, quien, si bien tras una larga interrupción de nuestra amistad, me mostró un corazón lleno de cariño» (NS IV, 187, línea 23-25); éste — como se ha dicho— siguió junto a Novalis las lecciones de Reinhold. Sin lugar a dudas, Forberg estaba tan influenciado por la formulación de Novalis con la que he comenzado la sección I de este texto, que escribiría un año más tarde en sus Cartas sobre la más novedosa filosofía:

Algo como un último porqué es, por lo tanto, un último fundamento originario [UrGrund] que debo buscar para satisfacer las exigencias de mi razón.

Pero, ¿y si fuese imposible encontrar un fundamento originario [...]? -

Entonces no se seguiría otra cosa que la completa insatisfacción de la exigencia de mi razón - el hecho de que, de forma infinita, la razón [...] debiese continuar su investigación sin nunca poder llegar a un final. El absoluto sería entonces nada más que la idea de una imposibilidad [...].

[Pero,] ¿es una meta menos meta por el hecho de ser inalcanzable? ¿Es la apariencia del cielo menos encantadora por el hecho de que siempre sea sólo apariencia? (Forberg 1797, 66 y s.)

Manfred Frank

Universität Tübingen manfred.frank@uni-tuebingen.de 


\section{ABREVIATURAS}

KA: Friedrich Schlegel, Kritische Ausgabe seiner Werke, editado por Ernst Behler, Paderborn/München/Wien/Zürich, 1958

KrV: Immanuel Kant, Kritik der reinen Vernunft, Primera edición (cit: A) 1781, segunda edición (cit: B) 1787. Uso la siguiente edición: Immanuel Kant, Theoretische Philosophie. Texte und Kommentar in 3 volúmenes, editado por Georg Mohr, Frankfurt am Main: Suhrkamp, 2004.

KSA: Friedrich Nietzsche, Kritische Studienausgabe, ed. por Giorgio Colli y Mazzino Montinari, München: dtv, 1998.

KU: Immanuel Kant, Kritike der Urteilskraft, $2^{\circ}$ edición 1793, in: Kant (1996), pp. 479-880.

NS: Novalis, Schriften. Die Werke Friedrich von Hardenberg, editado por Paul Kluckhohn y Richard Stuttgart: Johlhammer, 1960.

Phj Philosophisches Journal einer Gesellschaft Teutscher Gelehrten, ed. por Friedrich Immanuel Niethammer, Profesor de filosofía en Jena, Neu Strelitz: Michaelis, 1795, desde 1797 con la colaboración de Johann Gottlieb Fichte como coeditor en Jena y Leipzig.

SSD: Richard Wagner, Sämtliche Schriften und Dichtungen, Volksausgabe, 12 vol., Leipzig: Breitkopf y Härtel, o. J. [1991].

SW: Friedrich Wilhelm Joseph Schelling, Sämmtliche Werke, ed. por K. F. A. Schelling, Stuttgart: Cotta 1856-1864.

\section{BIBLIOGRAFÍA}

Baumgarten, Alexander Gottlieb (1761), Acroasis logica. In Christianum L. B. de Wolff, Halle: Hemmerde.

Bondeli, Martin (1995), Das Anfangsproblem bei Karl Leonhard Reinhold. Eine systematische und Entwicklungsgeschichte Untersuchung zur Pbilosophie Reinholds in der Zeit von 1789 bis 1803, Frankfurt am Main: Klostermann.

Danto, Arthur (1993), «Das Ende der Kunst», en: Die philosophische Entmündigung der Kunst, Munich: Fink, p. 109-146 [existe trad. española en ]

DiEZ, Immanuel Carl (1997), Briefwechsel und Kantische Schriften. Wissensbegründung in der Glaubenskrise Tübingen-Jena (1790-1792), ed. de Dieter Henrich, Stuttgart: KlettCotta.

ERHARD, Johann Benjamin (1796), Rezensionen von Schellings Ich-Schrift, publicada en el Allgemeine Literatur-Zeitung de Jena Nr. 319, el martes 11 de octubre de 1796, 89-91. Réplica de Schelling (del 26 de octubre del 1796) con la breve respuesta de Erhard en: Schellingiana Rariora, seleccionado e introducido por Luigi Pareyson, Torino: Bottega d'Erasmo, 1977, p. 55-59.

FEUERBACH, Paul Johann Anselm (1795), «Über die Unmöglichkeit eines ersten absoluten Grundsatzes der Philosophie», en: PbJ II/4, p. 306-322. 
FORBERG, Friedrich Karl (1797), «Briefe über die neueste Philosophie», en PhJ, I: Volumen sexto del cuaderno quinto, p. 44-88; II: ibid (séptimo volumen del cuaderno cuatro), p. 259-272.

FRANK, Manfred (1983), «Auf der Suche nach einem Grund. Über den Umschlag von Erkenntniskritik in Mythologie bei Musi», en: Karl-Heinz Bohrer, Mythos und Moderne, Frankfurt am Main: Suhrkamp, pp. 318-362.

- (1998), Unendliche Annährung. Die Anfänge der philosophischen Frübromantik, Frankfurt am

Main: Suhrkamp.

— (1998b), «Von der Grundsatz-Kritik zur freien Erfindung. Die ästhetische Wende in den

Fichte-Studien des Novalis», in: Athenäum. Jahrbuch für Romanik, 8ªño, pp. 75-95.

- (1999) Style in Philosophy (= Christian Gauss Lectures Princeton 1990), impreso en:

Metaphilosophy, ed. por Armen T. Marsobian: Oxford: Blackwell Publishers Ltd. 1999.

HEGEL, Georg Wilhelm Friedrich (1995), Ästhetik, con un ensayo introductorio de Georg

Lukács, ed. por Friedrich Bassenge, Berlin: Aufbau-Verlag.

HeNRICH, Dieter (1991), Konstellationen. Probleme und Debatten am Ursprung der idealistischen Philosophie (1789-1795), Stuttgart: Klett-Cotta.

- (2004), Grundlegung aus dem Ich. Untersuchung zur Vorgeschichte des Idealismus Tübingen - Jena (1790-1794), Frankfurt am Main: Suhrkamp.

HOFFBAUER, Johann Christoph (1810), Ueber die Analysis in der Philosophie; rein grössten Theils analytischer Verusch veranlasst durch die erste, diesen Gegensand betreffende Preisfrage der Königlichen Akademie der Wissenschaft zu Berlin Abhandlungen verwandten Inhalts, Halle: Hemmerde y Schwetschke.

JACOBI, Friedrich Heinrich (1787), Ueber die Lehre des Spinoza in Briefen an Herrn Moses Mendelssobn. Nueva edición ampliada, Breslau: Löwe. Nueva edición crítica en: Friedrich Heinrich Jacobi, Werke, Voll. 1,1 y 1,2 Schriften zum Spinozastreit, editado por Kalus Hammacher und Irmgard-Maria Piske, Hamburg, Stuttgart-BadCanstatt: Meiner und Fromman-Holzboog, 1989.

KÖHLER, Dietmar (1999), «Von Schelling zu Hitler? Anmerkungen zu Heideggers Schelling-Interpretation von 1936 und 1941», in: Istvan M. Fehér und Wilhelm G. Jacobs, Zeit und Freiheit: Schelling - Schopenhauer - Kierkegaard - Heidegger (= Akten der Fachtagung der Internationalen Schelling-Gessekschaft, Budapest, del 24 al 27 de abril de 1997), Budapest: Éthos Könyvek, pp. 201-213.

LuKÁCS, Georg (1954), Die Zerstörung der Vernunft. Der Weg des Irrationalismus von Schelling z. Hitler, Berlin: Aufbau-Verlag (también en Werke, 1962, Vol. 9, Neuwied: Luchterhand) [trad. esp. en: ]

Muslow, Martin, y Marcelo STAMM (Ed.) (2005), Konstellationsforschung, Frankfurt am Main: Suhrkamp.

Menand, Louis (2001), The Metaphysical Club, A Story of Ideas in America, Newe York: Farrar, Straus and Giroux.

Niethammer, Friedrich Immanuel (1795), «Von den Ansprüchen des gemeinen Verstandes an die Philosophie», in: PhJ I/1, pp. 1-45. 
REINHOLD, Kar Leonhard (1789), Versuch einer neuen Theorie des menschlichen Vorstellungsvermögen, Prag, Jena: C. Widmann \& J. M. Mauke.

- (1790), Beyträge zur Berichtigung bisheriger Mißverstädnisse der Philosophen. Primer volumen, Das Fundament der Elementarphilosophie betreffend, Jena: Mauke.

SARTRE, Jean-Paul (1943), L'être et le néant. Essai d'ontologie phénoménologique, Paris : Gallimard. SCHMID, Carl Christian Erhard (1791), Empirische Psychologie, Jena: Cröker, Erster Teil.

- (1792), Rezension von C. L. Reinholds Ueber das Fundament des philosophischen Wissens, en: [Jenaer] Allgemeine Literatur-Zeitung, Nr. 92 y 93 del 9 y 10 de abril de 1792, pp. 49-56 y 57-60.

STAMM, Marcelo R. (1992), «Mit der Überzeugung der Entbehrlichkeit eines höchsten und einzigen Grundsatzes...». Ein Konstellationsporträt um Fr. I. Niethammer «Philosophisches Journal einer Gesellschaft teutscher Gelehrten», manuscrito no publicado, Munich.

WEISs, Hermann F. (1991), «Eine Reise nach Thüringen im Jahre 1791. Zu einer unbeachteten Begegnung Karl Wilhelm Justis und Joseph Friedrich Engelschalls mit Schiller und Novalis», en: Zeitschrift für hessische Geschichte und Landeskunde 101, p. 43-56.

WitTGENSTEIn, Ludwig (1984), Vorlesungen 1930-1935. Cambridge 1930-1932. A partir de las notas de John King y Desmond Lee, ed. por Desmond Lee. Cambridge 19321935. A partir de las notas de Alice Ambrose y Margaret Macdonald, ed. por Alice Ambrose. Traducido por Joachim Schulte, Frankfurt am Main: Suhrkamp. 